\title{
柠檬酸溶胶-凝胶法制备的 $\mathrm{Ce}_{1-\mathrm{x}} \mathrm{Zr}_{x} \mathrm{O}_{2}$ : 结构及其氧移动性
}

\author{
叶青 王瑞璞徐柏庆*
}

(清华大学化学系, 有机光电子与分子工程教育部重点实验室, 分子催化与定向转化研究室, 北京 100084)

\begin{abstract}
摘要 采用 XRF、XRD、Raman、XPS、 $\mathrm{H}_{2}$-TPR 以及与氩离子刻蚀相结合的 XPS 等表征技术对柠檬酸溶胶-凝胶 法制备的 $\mathrm{Ce}_{1-x} \mathrm{Zr}_{x} \mathrm{O}_{2}(0 \leqslant x \leqslant 1)$ 样品的结构及其氧移动性进行了研究. 结果表明, $\mathrm{Ce}_{1-x} \mathrm{Zr}_{x} \mathrm{O}_{2}$ 样品的晶型结构对其 中氧的移动性有明显影响. 当 $x \leqslant 0.15$ 时, $\mathrm{Ce}_{1-x} \mathrm{Zr}_{x} \mathrm{O}_{2}$ 以立方 $\mathrm{CeO}_{2}$ 相 $\mathrm{Ce}-\mathrm{Zr}-\mathrm{O}$ 固溶体存在, 随着 $\mathrm{Zr}$ 含量的逐渐 增加, $\mathrm{CeO}_{2}$ 晶胞体积减小、氧空位浓度增加, 氧移动性逐渐增强; 当 $x>0.15$ 时, 形成四方 $\mathrm{ZrO}_{2}$ 相和立方 $\mathrm{CeO}_{2}$ 相 $\mathrm{Ce}-\mathrm{Zr}-\mathrm{O}$ 固溶体的混合物, 随着 $\mathrm{Zr}$ 含量的逐渐增加, 四方 $\mathrm{ZrO}_{2}$ 相的含量增加、氧空位浓度减小, 氧移动性逐渐减 弱. 因此, $\mathrm{Ce}_{0.85} \mathrm{Zr}_{0.15} \mathrm{O}_{2}$ 样品具有最高的氧移动性.
\end{abstract}

关键词： $\mathrm{Ce}_{1-x} \mathrm{Zr}_{x} \mathrm{O}_{2}$, 溶胶-凝胶法, 氧移动性, 氧空位, $\mathrm{CeO}_{2}$ 基固溶体

中图分类号: 0643

\section{Structure and Oxygen Mobility of $\mathrm{Ce}_{1-x} \mathrm{Zr}_{x} \mathrm{O}_{2}$ Prepared by Citric Acid Sol-gel Method}

\author{
YE, Qing WANG, Rui-Pu XU, Bo-Qing* \\ (Innovative Catalysis Program, Key Laboratory of Organic Optoelectronics \& Molecular Engineering, Department of Chemistry, \\ Tsinghua University, Beijing 100084, P. R. China)
}

\begin{abstract}
The structure and oxygen mobility of $\mathrm{Ce}_{1-1} \mathrm{Zr}_{x} \mathrm{O}_{2}(0 \leqslant x \leqslant 1)$ samples prepared by citric acid sol-gel method were characterized by XRF, XRD, Raman, XPS, $\mathrm{Ar}^{+}$etching-XPS and $\mathrm{H}_{2}$-TPR. The data showed that the crystalline structure of $\mathrm{Ce}_{1-x} \mathrm{Zr}_{x} \mathrm{O}_{2}$ can significantly affect the oxygen mobility. When $x \leqslant 0.15$, the samples were found to exist as cubic $\mathrm{Ce}-\mathrm{Zr}-\mathrm{O}$ solid solutions. An increase in $x$ resulted in a shrinking of the crystal unit volume, an increasing in the number of oxygen vacancies, and an increasing in the oxygen mobility as well. When $x>0.15, \mathrm{Ce}_{1-x} \mathrm{Zr}_{x} \mathrm{O}_{2}$ existed in a mixed phases of tetragonal and cubic $\mathrm{Ce}-\mathrm{Zr}$-O solid solutions; the percentage of tetragonal phase increased, oxygen vacancies decreased and oxygen mobility reduced with increasing $x$ in these samples. Therefore, this present work shows that oxygen mobility in the $\mathrm{Ce}_{0.85} \mathrm{Zr}_{0.15} \mathrm{O}_{2}$ sample is the highest.
\end{abstract}

Keywords: $\mathrm{Ce}_{1-x} \mathrm{Zr}_{x} \mathrm{O}_{2}$, Sol-gel method, Oxygen mobility, Oxygen vacancy, $\mathrm{CeO}_{2}$-based solid solution

自 20 世纪 90 年代以来, $\mathrm{CeO}_{2}$ 被广泛应用于各 种催化剂体系中, 其作用主要集中在: (1)促进贵金 属的分散性和稳定性 ${ }^{[1]} ;(2)$ 通过 $\mathrm{Ce}^{4+} / \mathrm{Ce}^{3+}$ 变换 $2 \mathrm{CeO}_{2}=$ $\mathrm{Ce}_{2} \mathrm{O}_{3}+1 / 2 \mathrm{O}_{2}$ (氧化气氛中为 $\mathrm{CeO}_{2}$, 还原气氛中为 $\left.\mathrm{Ce}_{2} \mathrm{O}_{3}\right)^{[2]}$, 表现出优良的储氧能力 (OSC), 有助于消除 汽车尾气中的 $\mathrm{NO}_{x} 、 \mathrm{CO}$ 和碳氢化合物 $(\mathrm{HC})$. 因此, $\mathrm{CeO}_{2}$
成为 $\mathrm{CO}$ 氧化 ${ }^{[3]}$, 水煤气变换(WGS)和三效催化转化 技术中的有效添加剂. 但由于 $\mathrm{CeO}_{2}$ 本身的热稳定性 较差, 容易烧结, 使其储氧性能受损 ${ }^{[4]}$, 限制了 $\mathrm{CeO}_{2}$ 的 应用效果, 因此提高其热稳定性和储氧能力具有重 要的意义. 人们发现在 $\mathrm{CeO}_{2}$ 中添加过渡金属和非 金属 ${ }^{[5-6]}$, 如: $\mathrm{Al}^{3+}, \mathrm{Si}^{4+}, \mathrm{Ti}^{4+}, \mathrm{Zr}^{4+}$, 可以改善 $\mathrm{CeO}_{2}$ 的热稳

Received: June 15, 2005; Revised: July 12, $2005 . \quad$ *Correspondent, E-mail: bqxu@ mail. tsinghua. edu. cn; Tel\&Fax: 010-62792122. 国家自然科学基金(20125310, 20590360)资助项目 
定性和储氧能力, 其中以 $\mathrm{Ce}_{1-\mathrm{X}} \mathrm{Zr}_{x} \mathrm{O}_{2}$ 为最好. 而且 $\mathrm{Ce}_{1-x} \mathrm{Zr}_{x} \mathrm{O}_{2}$ 本身对 $\mathrm{CO} 、 \mathrm{CH}_{4}$ 和 $\mathrm{C}_{2} \mathrm{H}_{6}$ 的催化氧化具有 较好的活性 ${ }^{[-11]}$. 有关 $\mathrm{CeO}_{2}-\mathrm{ZrO}_{2}$ 的氧化还原性和储 氧能力 (OSC) 的报道很多 ${ }^{[12-13]}$, 但对其氧移动性研 究较少, 而氧化物的氧移动性对催化氧化反应和抑 制催化剂失活起着重要的作用. 例如: Ce部分取代 $\mathrm{La}$ 形成具有高氧移动性的缺陷结构 $\mathrm{La}_{1-x} \mathrm{Ce}_{x} \mathrm{CoO}_{3}$, 可 明显提高甲烷催化燃烧反应活性 ${ }^{[14]}$. 有高氧移动性 的 $\mathrm{Ph} / \mathrm{Pr}-\mathrm{Ce}$ 对 $\mathrm{N}_{2} \mathrm{O}$ 分解反应的活性明显高于 $\mathrm{Rh} / \mathrm{CeO}_{2}$ [15]. $\mathrm{Ce}_{1-\mathrm{Z}} \mathrm{Zr}_{x} \mathrm{O}_{2}$ 的制备方法有很多, 如: 高能球磨法、 共沉淀法、溶胶-凝胶法和水热法等 ${ }^{[16-17]}$, 其中溶胶-凝 胶法能制得高均匀性样品. 本研究采用柠檬酸溶胶凝胶技术制备了系列 $\mathrm{Ce}_{1-x} \mathrm{Zr}_{x} \mathrm{O}_{2}$ 样品, 研究了它们的 组成、结构和氧移动性的关系.

\section{1 实验部分}

将不同比例的 $\mathrm{Ce}\left(\mathrm{NO}_{3}\right)_{3} \cdot 6 \mathrm{H}_{2} \mathrm{O}$ (分析纯, 北京新 华化学试剂厂) 和 $\mathrm{ZrO}\left(\mathrm{NO}_{3}\right)_{2} \cdot 2 \mathrm{H}_{2} \mathrm{O}$ (分析纯, 北京刘 李店化工厂)溶液加人到等摩尔的柠檬酸(分析纯, 北 京益利精细化学品有限公司) 溶液中, 得混合液. 将 混合液在 95 $100{ }^{\circ} \mathrm{C}$ 搅拌下蒸发至透明凝胶, 将凝胶 在 $120{ }^{\circ} \mathrm{C}$ 烘干, 然后再在 $800{ }^{\circ} \mathrm{C}$ 焙烧 $5 \mathrm{~h}$, 得到不同 组成 $\mathrm{Ce}_{1-\mathrm{x}} \mathrm{Zr}_{x} \mathrm{O}_{2}$ 样品.

采用日本岛津生产的 XRF-1700 型 X 苂光分析 仪测定样品组成, 用 $\mathrm{Rh}$ 靶作为激发源, $40 \mathrm{kV} 、 70$ $\mathrm{mA}$, 质量比为 $1: 1$ 纯 $\mathrm{CeO}_{2}$ 和 $\mathrm{ZrO}_{2}$ 为标定物, 结果 (表 1) 表明测得组成与投料组成相一致; XRD 测试 在 Bruker 公司 D8 型 X 射线粉末衍射仪上进行, $\mathrm{Cu}$ $K_{\alpha}$ 射线, $\mathrm{Ni}$ 滤光源, 光源波长为 $\lambda=0.15418 \mathrm{~nm}$, 扫描 速率为 $0.02\left(^{\circ}\right) \cdot \mathrm{s}^{-1}$, 管压 $40 \mathrm{kV}$, 管流 $30 \mathrm{~mA}$. Raman 光谱测定在 Renishaw 的 RM2000 型共焦显微拉 曼仪上进行, He-Ne 激光 $(623.8 \mathrm{~nm})$, 扫描范围为 50
$1000 \mathrm{~cm}^{-1}$, 扫描步长为 $1 \mathrm{~cm}^{-1} ; X$ 射线光电子能谱 (XPS)在AEM PH15300型X射线光电子能谱仪上进 行, $\mathrm{Al} K_{\alpha}$ 靶, 分辨率大于 $0.1 \mathrm{eV}$, 利用污染碳 $(\mathrm{C} 1 s$, $\left.E_{\mathrm{B}}=284.6 \mathrm{eV}\right)$ 为内标校正能量标尺, $1.33 \times 10^{-7} \mathrm{~Pa}$. 样品在测量前预先在 $650{ }^{\circ} \mathrm{C}$ 用 $\mathrm{CO}_{2}$ 处理 $20 \mathrm{~min} ; \mathrm{Ar}$ 离子刻蚀-XPS(Ar+刻蚀-XPS), 在 Leybold-HeraeusShenyang SKL-12 生产的 VG CLAM4 能谱仪上完 成. 样品测试采用 $\mathrm{Al} K_{\alpha}(1486.5 \mathrm{eV})$, 仪器腔内压强 $1.07 \times 10^{-7} \mathrm{~Pa}$. 采用 $\mathrm{AG} 21 \mathrm{Ar}^{+}$离子枪对样品做大束斑 刻蚀, 加速电压 $2 \sim 3 \mathrm{kV}$, 工作电流 $20 \mu \mathrm{A}$.

$\mathrm{H}_{2}-\mathrm{TPR}$ 测试在自制的程序升温还原装置上进 行, 热导池检测, 样品用量 $50 \mathrm{mg}$, 样品在 $\mathrm{Ar}$ 气中 $600{ }^{\circ} \mathrm{C}$ 预处理 $2 \mathrm{~h}$, 以脱除表面吸附的碳酸根 ${ }^{[18]}$, 降至 室温后, 切换流速为 $20 \mathrm{~mL} \cdot \mathrm{min}^{-1}$ 的 $5 \% \mathrm{H}_{2} / \mathrm{N}_{2}$ 混合 气, 以 $15{ }^{\circ} \mathrm{C} \cdot \mathrm{min}^{-1}$ 的速率升温至 $950{ }^{\circ} \mathrm{C}$, 然后保持 恒温 $30 \mathrm{~min}$.

\section{2 结果与讨论}

\section{$2.1 \mathrm{Ce}_{1-x} \mathrm{Zr}_{x} \mathrm{O}_{2}$ 的结构特点}

通常在温度低于 $1400{ }^{\circ} \mathrm{C}$ 时, $\mathrm{Ce}_{1-x} \mathrm{Zr}_{x} \mathrm{O}_{2}$ 可以立 方相、四方相、单斜相以及 $t 、 t^{\prime} 、 t^{\prime \prime}$ 亚稳相形式存在. 由 于阳离子 $\left(\mathrm{Ce}^{4+}\right.$ 和 $\left.\mathrm{Zr}^{4+}\right)$ 的扩散速率远小于氧离子 $\left(\mathrm{O}^{2-}\right)$ 的扩散速率 ${ }^{[19]}$, 文献中有关 $\mathrm{Ce}_{1-\mathrm{z}} \mathrm{Zr}_{x} \mathrm{O}_{2}$ 样品晶相组成 的数据差别较大. 我们采用 $\mathrm{XRD} 、$ Raman 和 $\mathrm{Ar}^{+}$刻 蚀-XPS 对 $\mathrm{Ce}_{1-x} \mathrm{Zr}_{x} \mathrm{O}_{2}$ 的晶相结构进行了表征.

\subsubsection{X-ray diffraction (XRD)}

图 1 和表 1 为 $\mathrm{XRD}$ 结果. 可以看出, $x \leqslant 0.15$ 时, 只出现立方 $\mathrm{CeO}_{2}$ 相(PDF34-0394), 相应的 $\mathrm{CeO}_{2}$ 相衍射峰从 $x=0$ 时的 $2 \theta \approx 28.6^{\circ}, 47.68^{\circ}$ 位移至 $x=$ 0.15 时的 $2 \theta \approx 29^{\circ}, 48^{\circ}$, 晶胞参数 “ $a$ ” 从 $0.5427 \mathrm{~nm}$ 减少到 $0.5391 \mathrm{~nm}$, 这是由于离子半径小于 $\mathrm{Ce}^{4+}$ $(0.094 \mathrm{~nm})$ 的 $\mathrm{Zr}^{4+}(0.086 \mathrm{~nm})^{[20]}$ 进人 $\mathrm{CeO}_{2}$ 晶格中, 使

表 $1 \mathrm{Ce}_{1-\mathrm{x}} \mathrm{Zr}_{x} \mathrm{O}_{2}$ 样品的组成、比表面积、O 1s-XPS 和还原特点

Table 1 Composition, surface area, O $1 s$-XPS and $\mathrm{H}_{2}$-TPR data of $\mathrm{Ce}_{1-1} \mathrm{Zr}_{x} \mathrm{O}_{2}$ samples

\begin{tabular}{|c|c|c|c|c|c|c|c|}
\hline \multirow{2}{*}{ Sample } & \multirow{2}{*}{$x^{*}$} & \multirow{2}{*}{$S_{\mathrm{BET}} /\left(\mathrm{m}^{2} \cdot \mathrm{g}^{-1}\right)$} & \multirow{2}{*}{$a / \mathrm{nm}$} & \multicolumn{3}{|c|}{ O $1 s$-XPS ** } & \multirow{2}{*}{$\frac{\mathrm{TPR}}{Y(\%)^{* * *}}$} \\
\hline & & & & $A_{1}$ & $A_{2}$ & $A_{2} / A_{1}$ & \\
\hline $\mathrm{ZrO}_{2}$ & 0.99 & 2.63 & - & 26141 & 12612 & 0.48 & - \\
\hline $\mathrm{Ce}_{0.2} \mathrm{Zr}_{0.8} \mathrm{O}_{2}$ & 0.80 & 3.00 & 0.5176 & 27416 & 23040 & 0.84 & 97.2 \\
\hline $\mathrm{Ce}_{0.5} \mathrm{Zr}_{0.5} \mathrm{O}_{2}$ & 0.51 & 17.1 & 0.5289 & - & - & - & 43.8 \\
\hline $\mathrm{Ce}_{0.75} \mathrm{Zr}_{0.25} \mathrm{O}_{2}$ & 0.25 & 32.1 & 0.5426 & 28620 & 30014 & 1.05 & 70.7 \\
\hline $\mathrm{Ce}_{0.25} \mathrm{Zr}_{0.15} \mathrm{O}_{2}$ & 0.16 & 34.3 & 0.5391 & 31823 & 36266 & 1.14 & 74.9 \\
\hline $\mathrm{Ce}_{0.95} \mathrm{Zr}_{0.05} \mathrm{O}_{2}$ & 0.06 & 23.1 & 0.5418 & 28446 & 28686 & 1.01 & 66.6 \\
\hline $\mathrm{CeO}_{2}$ & 0.00 & 8.90 & 0.5427 & 30850 & 21943 & 0.71 & 37.4 \\
\hline
\end{tabular}

$* x$ in $\mathrm{Ce}_{1-1} \mathrm{Zr}_{x} \mathrm{O}_{2}$ measured by XRF; ** $A_{1}$ and $A_{2}$ denote the XPS peak area shown in Fig.4; *** reduction percentage of $\mathrm{CeO}_{2}(\%)$ 


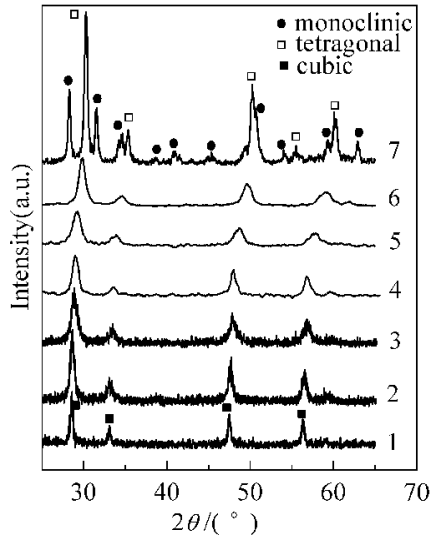

图 $1 \mathrm{Ce}_{1-x} \mathrm{Zr}_{x} \mathrm{O}_{2}$ 样品的 $\mathrm{X}$ 射线衍射图

Fig.1 XRD patterns of $\mathrm{Ce}_{1-x} \mathrm{Zr}_{x} \mathrm{O}_{2}$ samples

(1) $x=0$; (2) $x=0.05$; (3) $x=0.15$; (4) $x=0.25$; (5) $x=0.5$;

(6) $x=0.8$; (7) $x=1$

$\mathrm{CeO}_{2}$ 晶胞收缩所致, 表明此时形成了具有立方 $\mathrm{CeO}_{2}$ 相结构的Ce-Zr-O固溶体. 这与Roitti等 ${ }^{[21]}$ 的研究结 果相一致. 当样品中 $x>0.15$ 时, 衍射峰呈现不对称 性, 对峰形解叠表明在这些样品中同时存在四方 $\mathrm{ZrO}_{2}$ 相和立方 $\mathrm{CeO}_{2}$ 相, $\mathrm{CeO}_{2}$ 相的衍射峰从 $x=0.25$ 时的 $2 \theta \approx 29^{\circ}, 47.8^{\circ}$ 移至 $x=0.8$ 时的 $2 \theta \approx 29.8^{\circ}$, $49.6^{\circ}$, 晶格参数 “ $a$ ”从 $0.5426 \mathrm{~nm}$ 减少到 $0.5176 \mathrm{~nm}$. 表明此时的立方 $\mathrm{CeO}_{2}$ 相实际上也是 $\mathrm{Ce}-\mathrm{Zr}-\mathrm{O}$ 固溶 体相. 因此, $x>0.15$ 时, 样品为四方 $\mathrm{ZrO}_{2}$ 相和立方 $\mathrm{CeO}_{2}$ 相 Ce- $\mathrm{Zr}-\mathrm{O}$ 固溶体的混合物. 纯 $\mathrm{ZrO}_{2}(x=1)$ 以四 方相和单斜相 $\left(28.1^{\circ}\right.$ 和 $\left.31.3^{\circ}\right)$ 混合相存在.

\subsubsection{Raman 和 $\mathrm{Ar}^{+}$刻蚀-XPS}

XRD 技术对高分散和低浓度组分敏感性较低, 难以测定 $\mathrm{Ce}_{1-\mathrm{x}} \mathrm{Zr}_{x} \mathrm{O}_{2}$ 结构中可能存在的 $t 、 t^{\prime}$ 和 $t^{\prime \prime}$ 亚稳 相 ${ }^{[2]}$. 考虑到Raman技术对于 $\mathrm{M}-\mathrm{O}$ 伸缩有较好的敏

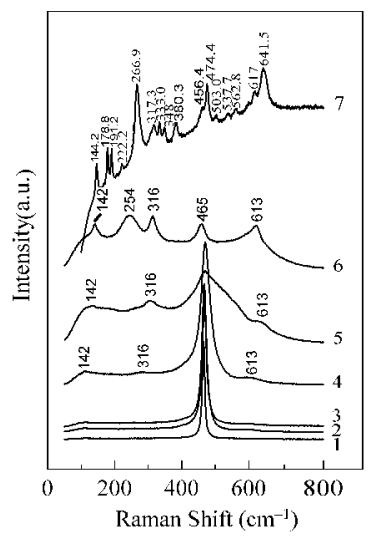

图 $2 \mathrm{Ce}_{1-x} \mathrm{Zr}_{x} \mathrm{O}_{2}$ 样品的 $\operatorname{Raman}$ 谱图

Fig.2 Raman spectra of $\mathrm{Ce}_{1-x} \mathrm{Zr}_{x} \mathrm{O}_{2}$ samples

(1) $x=0$; (2) $x=0.05$; (3) $x=0.15$; (4) $x=0.25$; (5) $x=0.5$;

(6) $x=0.8$; (7) $x=1$
感性 ${ }^{[23]}$, 我们对 $\mathrm{Ce}_{1-x} \mathrm{Zr}_{x} \mathrm{O}_{2}$ 样品的结构采用 Raman 技术进行了表征, 结果见图 2 . 可以看出, 当 $x \leqslant 0.15$ 时, 只在 $465 \mathrm{~cm}^{-1}$ 处显示了 $F_{2 \mathrm{~g}}$ 立方结构特征峰 $(F m 3 m$ 空间点群 $)^{[24]}$; 而在 $x>0.15$ 时, 不仅有 $465 \mathrm{~cm}^{-1}$ 处的立 方结构特征峰, 而且在 $142 、 254 、 316 、 455 、 590$ 和 613 $\mathrm{cm}^{-1}$ 处出现了与 $A_{1 \mathrm{~g}}+2 B_{\mathrm{lg}}+3 E_{\mathrm{g}}$ 振动有关的四方 $\mathrm{ZrO}_{2}$ 相 的Raman 特征峰(空间点群 $P 42 / n m c)^{[2]]}$. 图中未出现 $t 、 t^{\prime}$ 和 $t^{\prime \prime}$ 亚稳相 ${ }^{[19]}$. 纯 $\mathrm{ZrO}_{2}(x=1)$ 中只存在四方相(146、 $\left.266 、 317 、 333 、 456 、 474 、 641 \mathrm{~cm}^{-1}\right)$ 和单斜相 $(178 、 191$ 、 $222 、 348 、 503 、 537 、 562 、 617 \mathrm{~cm}^{-1}$ ), 不存在立方相, 这 与 XRD和 $\mathrm{Ce}_{1-x} \mathrm{Zr}_{x} \mathrm{O}_{2}$ 相图 ${ }^{[22]}$ 结果相一致.

为进一步验证以上结果, 对 $\mathrm{Ce}_{1-\mathrm{X}} \mathrm{Zr}_{x} \mathrm{O}_{2}$ 进行了 $\mathrm{Ar}^{+}$刻蚀-XPS 分析, 结果见图 3. 由图 3 可以看出, 对 于 $\mathrm{Ce}_{0.2} \mathrm{Zr}_{0.8} \mathrm{O}_{2}$, 随着刻蚀时间增加, $n_{\mathrm{zr}} / n_{\mathrm{ce}}$ 比值迅速 减少, 然后基本保持稳定, 表明此样品的表面“浅层” 部分 $\mathrm{ZrO}_{2}$ 较多, 而深层部分 $n_{\mathrm{Zr}} / n_{\mathrm{Ce}}$ 比变化较小表明 形成了均匀的 Ce- $\mathrm{Zr}-\mathrm{O}$ 固溶体. 对于 $\mathrm{Ce}_{0.5} \mathrm{Zr}_{0.5} \mathrm{O}_{2}, n_{\mathrm{Zr}}$ I $n_{\mathrm{Ce}}$ 比只是在开始部分缓慢减少, 然后保持稳定, 表 明此时 $\mathrm{ZrO}_{2}$ 较少, 样品主要以 $\mathrm{Ce}-\mathrm{Zr}-\mathrm{O}$ 固溶体形式 存在. 对于 $\mathrm{Ce}_{0.85} \mathrm{Zr}_{0.15} \mathrm{O}_{2}, n_{\mathrm{Zr}} / n_{\mathrm{Ce}}$ 比例随着刻蚀时间增 加保持不变, 表明 $\mathrm{Ce}$ 和 $\mathrm{Zr}$ 分布均匀, 它们完全形 成了 Ce-Zr-O 固溶体, 这与 XRD 和 Raman 的结果 相一致.

\section{$2.2 \mathrm{Ce}_{1-x} \mathrm{Zr}_{x} \mathrm{O}_{2}$ 的氧移动性}

\subsubsection{1 s-XPS}

由于氧在 $\mathrm{CeO}_{2}$ 中的扩散是空位机理 ${ }^{[26]}$, 所以氧空 位与氧的移动性密切相关, 而氧空位可以通过O $1 s-$ $\mathrm{XPS}$ 检测. 图4为 $\mathrm{Ce}_{1-\mathrm{x}} \mathrm{Zr}_{\mathrm{x}} \mathrm{O}_{2}$ 的O $1 s$-XPS谱图, 由图可 见, O $1 s$-XPS由双峰组成, 结合能较低的峰 $\left(E_{\mathrm{b}}=529.5\right.$

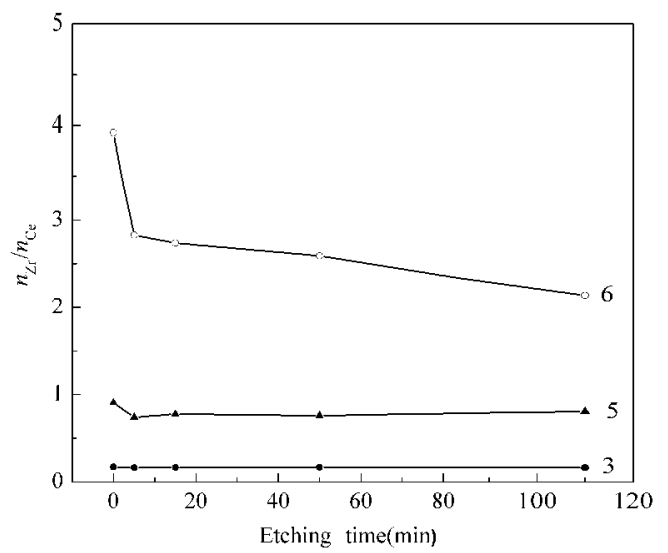

图 3 不同样品的 $\boldsymbol{n}_{\mathrm{Zr}} / \boldsymbol{n}_{\mathrm{Ce}}$ 比随 $\mathbf{A} \mathbf{r}^{+}$刻蚀时间的变化情况

Fig.3 Curves of $n_{\mathrm{Zr}} / n_{\mathrm{Ce}} v s \mathrm{Ar}^{+}$-etching time of $\mathrm{Ce}_{1-x} \mathrm{Zr}_{x} \mathbf{O}_{2}$ samples

(3) $x=0.15$; (5) $x=0.5$; (6) $x=0.8$ 


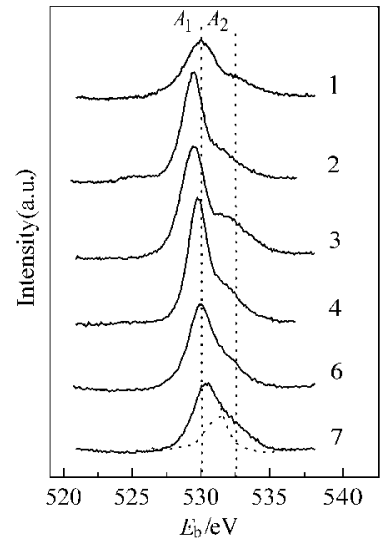

图 $4 \mathrm{Ce}_{1-x} \mathrm{Zr}_{x} \mathrm{O}_{2}$ 样品的 $01 s-X P S$ 谱图

Fig.4 0 1s-XPS spectra of $\mathrm{Ce}_{1-x} \mathrm{Zr}_{x} \mathrm{O}_{2}$ samples

(1) $x=0$; (2) $x=0.05$; (3) $x=0.15$; (4) $x=0.25$; (6) $x=0.8$;

(7) $x=1$

$\mathrm{eV})$ 为表面晶格氧, 结合能较高的峰 $\left(E_{\mathrm{b}}=532.3 \mathrm{eV}\right)$ 为吸附氧 (吸附水的 $-\mathrm{OH}^{[27]}$ 和 $\mathrm{CO}_{3}^{2-}$ 的 $\mathrm{C}-\mathrm{O}^{[27]}$ ). 因为 $\mathrm{Ce}_{1-x} \mathrm{Zr}_{x} \mathrm{O}_{2}$ 样品在测量前已用 $\mathrm{CO}_{2}$ 在 $650{ }^{\circ} \mathrm{C}$ 处理, 所以应该没有吸附水的XPS 信号. 表 1 给出了由高 斯解析得到的晶格氧 $\left(A_{1}\right)$ 和吸附氧 $\left(A_{2}\right)$ 的 XPS 面积 结果. Palmqvist 等 ${ }^{[23]}$ 认为吸附氧的 XPS 峰源于氧 空位捕获的碳酸根物种, 通过比较吸附氧的 XPS 峰 强度可以比较氧空位的量. 当 $x \leqslant 0.15$ 时, $\mathrm{Ce}_{1-x} \mathrm{Zr}_{x}-$ $\mathrm{O}_{2}$ 以立方结构存在, 随 $x$ 逐渐减小, 吸附氧峰面积 $A_{2}$ (或 $A_{2} / A_{1}$ 比值)逐渐降低, 即氧空位浓度降低; 当 $x$ $>0.15$ 时, $\mathrm{Ce}_{1-x} \mathrm{Zr}_{x} \mathrm{O}_{2}$ 以立方和四方相的混合物存在, 随着 $x$ 减少, 吸附氧面积 $A_{2}$ 或 $\left(A_{2} / A_{1}\right)$ 增加, 即氧空位 增多. 因此, $\mathrm{Ce}_{0.85} \mathrm{Zr}_{0.15} \mathrm{O}_{2}$ 样品具有最高的吸附氧峰面 积 $A_{2}$ (或最高的 $A_{2} / A_{1}$ 比值), 表明其氧空位最多.

\subsection{2 $\mathrm{H}_{2}$-TPR}

图 5 为 $\mathrm{Ce}_{1-\mathrm{x}} \mathrm{Zr}_{x} \mathrm{O}_{2}$ 的 $\mathrm{H}_{2}-\mathrm{TPR}$ 结果. 纯 $\mathrm{ZrO}_{2}(x=1)$ 本身没有还原峰(图中未显示), 纯 $\mathrm{CeO}_{2}(x=0)$ 的 TPR 谱线上出现了 $\mathrm{Ce}^{4+} / \mathrm{Ce}^{3+}$ 两个还原峰, 峰温分别为 550 和 $880{ }^{\circ} \mathrm{C}$; 其中的低温峰归属为表层 $\mathrm{Ce}^{4+} / \mathrm{Ce}^{3+}$ 的 还原, 高温峰归属为体相 $\mathrm{Ce}^{4+} / \mathrm{Ce}^{3+}$ 的还原 ${ }^{[3]}$. 有趣的 是, TPR 上出现的还原峰数目与样品中 $\mathrm{Zr}$ 浓度密切 相关, 当 $x<0.5$ 时, 在 $548 \sim 640{ }^{\circ} \mathrm{C}$ 和 $741 \sim 850{ }^{\circ} \mathrm{C}$ 存 在两个还原峰; $x \geqslant 0.5$ 时, 只存在一个低温还原峰, 表明此时表面和体相中的 $\mathrm{Ce}^{4+}$ 同时被还原. 样品中 $\mathrm{Ce}^{4+}$ 还原程度随 $\mathrm{Zr}$ 浓度增加而增加, 从纯 $\mathrm{CeO}_{2}$ 的 $37.4 \%$ 增加到 $\mathrm{Ce}_{0.2} \mathrm{Zr}_{0.8} \mathrm{O}_{2}$ 的 $97.2 \%$.

TPR 谱线上的还原峰温度的高低表征了样品 中氧的移动性, 还原峰温越低说明氧的移动性越高.

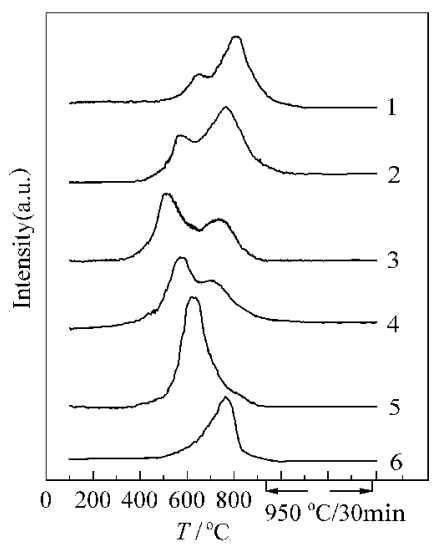

图 $5 \mathrm{Ce}_{1-x} \mathrm{Zr}_{x} \mathrm{O}_{2}$ 样品的 TPR 谱图

Fig.5 TPR profiles of $\mathrm{Ce}_{1-x} \mathrm{Zr}_{x} \mathrm{O}_{2}$ samples

(1) $x=0$; (2) $x=0.05$; (3) $x=0.15$; (4) $x=0.25$; (5) $x=0.5$;

(6) $x=0.8 .950{ }^{\circ} \mathrm{C} / 30 \mathrm{~min}$ on the abscissa indicates that the temperature was kept constant at $950{ }^{\circ} \mathrm{C}$ for $30 \mathrm{~min}$.

$\mathrm{Ce}_{1-1} \mathrm{Zr}_{x} \mathrm{O}_{2}$ 低温还原峰温 $(\mathrm{LT})$ 和高温还原峰温 $(\mathrm{HT})$ 与 $\mathrm{Zr}$ 浓度和样品结构密切相关. 立方相固溶体结 构的 $\mathrm{Ce}_{1-x} \mathrm{Zr}_{x} \mathrm{O}_{2}$ 样品 $(x \leqslant 0.15)$ 随着 $\mathrm{Zr}$ 浓度增加, $\mathrm{LT}$ 和 HT 均向低温方向移动, 并在以 $x=0.15$ 时移动最 大, 即氧移动性随 $x$ 增加而增大. 对于四方和立方相 混合的 $\mathrm{Ce}_{1-1} \mathrm{Zr}_{x} \mathrm{O}_{2}$ 样品 $(x>0.15)$ 随 $\mathrm{Zr}$ 浓度增加, LT 逐渐向 $\mathrm{HT}$ 靠拢, 并最初终变为一个高温峰, 清楚地 表明氧的移动性随 $x$ 增大而降低. 因此, 在本研究中 $\mathrm{Ce}_{0.85} \mathrm{Zr}_{0.15} \mathrm{O}_{2}$ 表现出最低的 LT 和 HT, 即具有最高氧 移动性.

对于立方结构 $\mathrm{Ce}_{1-x} \mathrm{Zr}_{x} \mathrm{O}_{2}$, 随着 $\mathrm{Zr}$ 含量的增加, 氧 移动性增强, 这可能是因为离子半径较小的 $\mathrm{Zr}^{4+}$ 部分 取代 $\mathrm{Ce}^{4+}$, 缩小了 $\mathrm{CeO}_{2}$ 的晶胞体积, 产生了缺陷, 使 氧空位增加(图4)的结果, 由于氧在立方 $\mathrm{CeO}_{2}$ 中的扩 散符合空位机理 ${ }^{[2]]}$, 所以随着 $\mathrm{Zr}$ 含量的逐渐增加, 氧 离子迁移能减少, 有利于氧的移动. Balducci等 ${ }^{[29]}$ 的 理论模型结果表明, 在立方相结构的 $\mathrm{Ce}_{1-x} \mathrm{Zr}_{x} \mathrm{O}_{2}$ 中, $\mathrm{Zr}^{4+}$ 的存在能有效地降低氧移动的活化能, 且随着 $\mathrm{Zr}^{4+}$ 的增多活化能呈线性下降. 对于混合晶相结构的 $\mathrm{Ce}_{1-\mathrm{Z}} \mathrm{Zr}_{x} \mathrm{O}_{2}$, 氧移动性则是随 $\mathrm{Zr}$ 含量的增加而减弱. Fair研究了B-Si体系 ${ }^{[30]}$ 和Power等研究了多晶相 $\beta$ $\mathrm{Al}_{2} \mathrm{O}_{3}$ 体系 ${ }^{[3]}$, 认为对于多晶混合型化合物, 由于颗粒 边界存在多相异性, 氧在边界的扩散是氧移动的控 制步骤, 所以对于混合晶型结构的 $\mathrm{Ce}_{1-\mathrm{x}} \mathrm{Zr}_{x} \mathrm{O}_{2}$, 随着四 方结构成分的增加, 多相异性增加, 氧移动的活化能 增加, 而且另一方面, 在混合晶相的 $\mathrm{Ce}_{1-1} \mathrm{Zr}_{x} \mathrm{O}_{2}$ 样品 体系中, 氧空位浓度随 $\mathrm{Zr}$ 含量增加而减少 (图 4), 也 
导致了氧的移动性降低.

\section{3 结 论}

(1) 采用柠檬酸溶胶-凝胶法制备的 $\mathrm{Ce}_{1-\mathrm{x}} \mathrm{Zr}_{x} \mathrm{O}_{2}$ 中, 当 $x \leqslant 0.15$ 时, $\mathrm{Ce}$ 和 $\mathrm{Zr}$ 形成立方 $\mathrm{CeO}_{2}$ 相 $\mathrm{Ce}-\mathrm{Zr}-\mathrm{O}$ 固溶体; 当 $x>0.15$ 时, $\mathrm{Ce}$ 和 $\mathrm{Zr}$ 形成四方 $\mathrm{ZrO}_{2}$ 和立 方 $\mathrm{CeO}_{2}$ 相 $\mathrm{Ce}-\mathrm{Zr}-\mathrm{O}$ 固溶体的混合物.

(2) $\mathrm{Ce}_{1-x} \mathrm{Zr}_{x} \mathrm{O}_{2}$ 的O $1 s-\mathrm{XPS}$ 和 $\mathrm{H}_{2}-\mathrm{TPR}$ 结果表明, $x \leqslant 0.15$ 时, 随着 $\mathrm{Zr}$ 含量的增加, 氧空位和氧移动 性增加; $x>0.15$ 时, 随着 $\mathrm{Zr}$ 含量增加, 氧空位和氧 移动性降低. $\mathrm{Ce}_{0.85} \mathrm{Zr}_{0.15} \mathrm{O}_{2}$ 样品中的氧移动性为最高.

\section{References}

1 Harrison, B.; Diwell, A. F.; Hallett, C. Platinum Metals Rev., 1988, 32: 73 .

2 Yao, H. C.; Yu Yao, Y. F. J. Catal., 1984, 86: 254.

3 Oh, S. H.; Eickel, C. C. J. Catal., 1988, 112: 543.

4 Schmieg, S. J.; Belton, D. N. Appl. Catal. B, 1995, 6: 127

5 Liu, Y.; Zhong, B.; Peng, S. Y.; Wu, D., Fan, Y. Z.; Fan, W. H. Acta Phys.-Chim. Sin., 1996, 12(3): 265 [刘 源, 钟 炳, 彭少 逸, 吴 东, 樊彦贞, 范文浩. 物理化学学报 (Wuli Huaxue Хиebao), 1996, 12(3): 265]

6 Shangguan, R. C.; Ge, X.; Zhang, H. L. Acta Phys. -Chim. Sin., 1999, 15(6): 568 [上官荣昌, 葛 欣, 张惠良, 物理化学学报 (Wuli Huaxue Xиebao), 1999, 15(6): 568]

7 Li, C. L.; Fu, Y. L.; Bian, G. Z. Acta Phys.-Chim. Sin., 2003, 19: 902 [李春林, 伏义路, 市国柱. 物理化学学报 (Wuli Huaxue Хиebao), 2003, 19: 902]

8 Chen, K. D.; Yan, Q. J. Acta Phys.-Chim. Sin., 1996, 12: 990 [陈开东, 颜其洁. 物理化学学报(Wuli Huaxue Xuebao), 1996, 12: 990]

9 Zhang, J. J.; Liu, Y. J.; Li, N.; Lin, B. X. Acta Phys.-Chim. Sin., 1999, 15: 15 [张继军, 刘英俊, 李 能, 林炳雄. 物理化学学报 (Wuli Ниахие Хиеbao), 1999, 15: 15]

10 Li, C. L.; Fu, Y. L.; Tu, K. Chinese Journal of Catalysis, 2004, 25 (6): 450 [李春林, 伏义路, 屠 兢. 催化学报(Cuihua Xuebao), 2004, 25(6): 450]

11 Wang, W. D.; Lin, P. Y.; Meng, M.; Hu, T. D.; Xie, Y. N.; Liu, T. Journal of the Chinese Rare Earth Society, 2002, 20(3): 265 [汪文栋, 林培琰, 孟 明, 胡天斗, 谢亚宁, 刘 涛. 中国稀土学 报(Zhongguo Xitu Xuebao), 2002, 20(3): 265]

12 Xu, D. P.; Wang, Q. Y.; Zhang, G. M.; Li, L. P.; Su, W. H.
Chemical Journal of Chinese Universities, 2001, 22(4): 524 [许大鹏, 王权泳, 张弓木, 李莉萍, 苏文辉. 高等学校化学学报 (Gaodeng Xuexiao Huaxue Xиebao), 2001, 22(4): 524]

13 Fornasiero, P.; Di Monte, R.; Ranga Rao, G.; Kaspar, J.; Meriani, S.; Trovarelli, A.; Graziani, M. J. Catal., 1995, 151: 168

14 Kirchnerova, I.; Alifanti, M.; Delmon, B. C. Appl. Catal. A, 2002, 231: 65

15 Imamura, S.; Tadani, J.; Saito, Y.; Okamoto, Y.; Jindai, H.; Kaito, C. Appl. Catal. A, 2000, 201: 121

16 Feng, C. G., Zhang, J. S., Wang, Y. J. Chinese Journal of the Ceramic Society, 2004, 32 (4): 502 [冯长根, 张江山, 王亚军. 硅酸盐学报(Guisuanyan Xuebao), 2004, 32(4): 502]

17 Luo, M. F.; Zhou, B.; Lin, R.; Chen, M.; Yuan, X. X.; Zheng, X. M. Chinese Journal of Rare Earths, 2000, 18(4): 275 [罗孟飞, 周 碧, 林 瑞, 陈 敏, 袁贤金鍂, 小明. 稀土化学学报 (Xitu Huaxue Xиebao), 2000, 18(4): 275]

18 Laachir, A.; Perrichon, V.; Badri, A.; Lamotte, J.; Catherine, E.; Lavalley, J. C.; Fallah, J. El; Hilaire, L.; Normand, F. Le; Quemere, E.; Sauvion, G. N. ; Touret, O. J. Chem. Soc., Faraday Trans., 1991, 87: 1601

19 Stubican, V. S.; Hellmann, J. R. Phase equilibria in some zirconia systems. Columbus: The American Ceramic Society, 1981: 25-36

20 Shannon, R. D. Acta Crystallogr. A, 1976, 32: 751

21 Roitti, S.; longo, V. Ceramurgia Int., 1972, 2(2): 97

22 McHale, A. E. Phase Diagrams for Ceramists Annual, 1991: 20

23 Knozinger, H.; Mestl, G. Top. Catal., 1999, 8: 45

24 Lin, X. M.; Li, L. P.; Li, G. S.; Su, W. H., Mater. Chem. Phys. 2001, 69: 236

25 Lopez, E. F.; Escribano, V. S.; Panizza, M.; Carnasciali, M. M.; Busca, G. J. Mater. Chem., 2001, 11: 1891

26 Kofstad, P. Nonstoichiometry, diffusion, and electrical conductivity in binary oxides. New York: Wiley, 1972

27 Moulder, J.; Stickle, W. F.; Sobol, P. E.; Bomben, K. D. Handbook of X-ray photoelectron spectroscopy. 2nd. Eden Prairie: PerkinElmer Corporation, 1992

28 Palmqvist, A. E. C.; Wirde, M.; Gelius U.; Muhammed, M. NanoStructured Materials, 1999, 11(8): 995

29 Balducci, G.; Kaspar, J.; Fornasiero, P.; Graziani, M.; Islam, M. S.; Gale, J. D. J. Phys. Chem. B, 1997, 101(10): 1750

30 Fair, R. B. J. Electrochem. Soc., 1975, 122: 800

31 Power, R. W.; Mitoff, S. P. Solid electrolytes general principles, characterization, materials, application. New York: Academic Press, 1978: Chap. 9 